NOTA CIENTÍFICA

\title{
MODELAGEM DO CRESCIMENTO FLORESTAL CONSIDERANDO VARIÁVEIS DO AMBIENTE: REVISÃO
}

\section{FOREST GROWTH MODELING TAKING INTO ACCOUNT ENVIRONMENTAL VARIABLES: A REVIEW}

\author{
Romualdo MAESTRI ${ }^{1}$ \\ Carlos Roberto SANQUETTA ${ }^{2}$ \\ José Roberto SCOLFORO ${ }^{3}$ \\ Sebastião do Amaral MACHADO ${ }^{4}$ \\ Ana Paula Dalla CORTE ${ }^{5}$
}

\begin{abstract}
RESUMO
Este trabalho teve como objetivo realizar uma revisão do estado da arte sobre a modelagem do crescimento florestal, através da inclusão de variáveis do ambiente. Os modelos visam expressar, de forma simplificada, algum aspecto da realidade. Na área florestal, os modelos de crescimento e produção são muito utilizados visando prever o comportamento dos mesmos, frente as mais diversas situações, subsidiando com informações, o gestor florestal. Em geral, a modelagem florestal descritiva, é baseada em variáveis independentes como: idade, sítio e densidade populacional. Existe uma tendência recente em incluir variáveis ambientais nos modelos de forma a proporcionar prognoses do crescimento e produção mais confiáveis.

Palavras-chave: variáveis ambientais; modelagem do crescimento; modelos.
\end{abstract}

\section{ABSTRACT}

This paper presents a review on the state-of-the-art of forest growth modeling that takes into account environmental variables. Models tries to express, in a simplified way, the reality. In forestry, models are very useful to predict the future development of the forests and stands, under various scenarios, providing information for decision-making by managers. In general, descriptive forest modeling is based upon independent variables such as: age, site index, and population density. There is a recent trend to include environmental variables in the models in order to provide more reliable growth and yield predictions. Key-words: environmental variables; growth modeling; models.

\footnotetext{
${ }^{1}$ M. Sc., Dr., Engenheiro Florestal, Granflor - Gestão de Empreendimentos Florestais Ltda, Porto Alegre, RS, Brasil, E-mail: rm@granflor.com.br

${ }^{2}$ M. Sc., Ph.D., Professor do Departamento de Ciências Florestais, UFPR, Lothário Meissner, 900, Jardim Botânico, CEP 80.210-170, Curitiba PR, Brasil. Pesquisador do CNPq. E-mail: sanquetta@ufpr.br. Autor correspondente.

${ }^{3}$ M.Sc., Dr. Professor do Departamento de Ciências Florestais, UFLA. Lavras, MG, Brasil. E-mail: scolforo@ufla.br

${ }^{4}$ M.Sc., PhD., Professor do Departamento de Ciências Florestais, UFPR, Curitiba, PR, Brasil, E-mail: samachado@ufpr.br

${ }^{5}$ M.Sc., Dr., Professor do Departamento de Ciências Florestais, UFPR, Lothário Meissner, 900, Jardim Botânico, CEP 80210-170, Curitiba, PR, Brasil. E-mail: anacorte@ufpr.br
} 
MAESTRI, R. et al. Modelagem do crescimento...

\section{INTRODUÇÃO}

Um modelo é uma abstração ou uma representação simplificada de algum aspecto da realidade. Modelo de crescimento e produção florestal é uma ferramenta matemática para representação da dinâmica natural de um povoamento florestal e modelagem é o processo de desenvolvimento dessa representação. A simulação de crescimento de uma árvore ou povoamento permite análises de seu comportamento sob determinadas condições, e assim, auxiliam as tomadas de decisão sobre seu melhor uso. O processo de desenvolvimento de modelos, por sua vez, é um pré-requisito da simulação, pois com base nas curvas esperadas de produção em função de variáveis independentes, a definição de uma opção mais adequada de manejo dentre as diversas possíveis, é viabilizada. O planejamento de uso dos recursos existentes dentro de um ambiente florestal também é efetuado tendo como base, modelos de crescimento e produção. Por outro lado, o entendimento dos processos de crescimento e das variáveis que afetam o desenvolvimento das árvores, quando considerados na modelagem, dotam as ferramentas de um sentido mais generalista, pois modelar a "causa" implica garantir que as hipóteses biológicas sejam consideradas e extrapolações mostram-se conceitualmente mais seguras. O desenvolvimento científico tem na área de modelagem florestal têm apresentado duas principais linhas filosóficas de abordagem do tema: modelos orientados ao manejo, chamados de modelos descritivos, empíricos ou estatísticos, e os modelos baseados em processos biológicos. Os cientistas envolvidos no desenvolvimento de cada linha de abordagem normalmente trabalham separadamente, mas muitos especialistas vislumbram uma tendência futura de maior embasamento ecológico dos modelos descritivos e de maior aplicabilidade prática dos modelos processuais, ou seja, ferramentas híbridas são esperadas nessa área. As principais virtudes de um bom modelo estão na sua capacidade de serem genéricos, realísticos e precisos, mas os modelos baseados em cada uma das filosofias (descritivos ou processuais), dificilmente apresentam-se com os três quesitos atendidos ao mesmo tempo. A inclusão de variáveis ambientais nos modelos de crescimento descritivos é uma forma concreta de evolução nesse sentido, pela melhoria da sua condição de generalidade.

\section{MODELOS BASEADOS EM PROCESSOS}

Modelos baseados em processos são também chamados de modelos mecanísticos ou modelos ecofisiológicos. Eles tratam de estimar o crescimento em volume, peso ou carbono de uma formação vegetal florestal em função do meio ambiente e de aspectos físicos ou ecológicos por incluir processos ou funções biológicas básicas na sua formulação, dotando-os de maior habilidade generalista.

Ao invés de serem desenvolvidos como modelos preditivos para suportar decisões de manejo, os modelos mecanísticos objetivam modelar os processos de crescimento tendo como variáveis independentes: luz, água, temperatura e níveis de nutrientes disponíveis no solo e, fornecem respostas em termos da alocação de biomassa em raízes, troncos e folhas através da modelagem da fotossíntese e da respiração (Landsberg, 1997). Os modelos processuais são úteis para compreender e ligar partes previamente isoladas do conhecimento e podem ajudar a identificar lacunas onde mais esforços de pesquisas se fazem necessários (Vanclay, 1999). Segundo Passioura (1996), os modelos mecanísticos podem ser chamados de modelos científicos, pois objetivam melhorar nosso entendimento sobre as interações da fisiologia e o do ambiente que afetam as plantações. Algumas vezes, segundo esse autor, eles têm falhado por serem baseados em suposições não testadas sobre os processos que controlam o crescimento e, nesse caso, tornamse úteis para o auto-aprendizado de seus propositores.

Muitos autores argumentam que a aplicação limitada dos modelos processuais como ferramenta prática é uma consequência do grande número de parâmetros necessários, da complexidade dos modelos e da falta de documentação apropriada. Mas por outro lado, o uso de modelos baseados em processos melhor contribui para a compreensão de como os fatores ambientais afetam o crescimento e podem auxiliar na definição da produtividade potencial em áreas sem histórico de cobertura florestal e sob diferentes condições ambientais (Almeida et al., 2002).

Um aspecto importante e que caracteriza um modelo processual na sua essência, é seu caráter de retro-alimentação ("feedback"), ou seja, o próprio resultado do modelo interfere nas variáveis independentes (do ambiente), influenciando uma nova inferência. E assim, poucos são os modelos disponíveis que possam ser caracterizados como puramente baseados em processos. Reed (1997) argumenta que todos os modelos são conceitualmente empíricos, uma vez serem baseados em dados de observações experimentais.

A questão da escala de tempo dos processos modelados tem impacto relevante nas respostas do modelo. Por exemplo, o efeito de um gás poluente na produção florestal tal como o ozônio, requer consideração do tempo, duração e intensidade de exposição em combinação com a suscetibilidade da planta através dos mecanismos de sequestro dos gases e aberturas dos estômatos. Modelos hábeis para estimar essa situação são muito complexos para examinar questões como idade de rotação ou densidade ótima de plantio (Reed, 1997). Segundo o mesmo autor, a escala temporal ideal de uso dos modelos não deve ultrapassar de duas a três vezes a magnitude do processo mais crítico representado no modelo. Tentativas de operar num passo de tempo e solicitar estimativas para escalas de diversas ordens dessa magnitude, quase sempre levam a resultados insatisfatórios.

Um termo técnico muito usado para parametrização dos coeficientes dos modelos ecofisioló- 
gicos é a "calibração". Uma vez que os fenômenos são previamente baseados em hipóteses, modelos matemáticos que assumam tais comportamentos são considerados fixos e seus coeficientes, calibrados. Segundo Jones et. al. (1998), calibração refere -se ao ajuste dos parâmetros do modelo de forma a permitir a "mais acurada" comparação entre o resultado da simulação e o resultado obtido de medições de campo. Por exemplo, em se supondo que a taxa fotossintética é uma função do material genético (ex. clones de eucalipto) e, considerando que todas as demais funções mantêm-se inalteradas, é possível através de calibração se determinar um modificador apropriado para esse parâmetro, através da comparação das simulações contra dados observados. Segundo os autores, em muitos casos, a calibração é o único meio prático de se estimar os valores dos parâmetros usados em modelos processuais de crescimento. Esse aspecto se assemelha ao processo de desenvolvimento dos modelos descritivos onde os métodos de "calibração" apoiam-se na análise de regressão estatística, porém a busca de modelos alternativos sempre caracterizou a filosofia de modelos biométricos, o que já não é uma prática usual no desenvolvimento dos modelos mecanísticos. Ambos os casos levam ao maior vínculo com a base de dados de ajuste e, os resultados tornam-se válidos para dentro dos limites originais, e assim, precisam estar fundamentados numa ampla cobertura de dados da situação em análise. O processo de validação contra uma base de dados independente sempre é uma garantia de sucesso das estimativas por parte de qualquer modelo, mas nesse caso em particular, torna-se fundamental.

\section{MODELOS DESCRITIVOS}

Modelos de crescimento e produção descritivos são também chamados de empíricos ou estatísticos, pois se baseiam em funções ajustadas a dados observados de uma amostra da população de interesse. Segundo Burkhart (1997), o termo "empírico" normalmente é associado à imagem de uma metodologia expedita e de menor qualidade, porém isso não representa a realidade, pois se tratam de modelos profundamente práticos e muito úteis para descrever situações do mundo real e, portanto, aplicáveis para a resolução de inúmeros de seus problemas.

Os modelos de crescimento auxiliam pesquisadores e manejadores em diversas formas. Vanclay (1999) ressalta a habilidade dos modelos em predizer produções futuras, o que permite uma eficiente previsão de uso de recursos, mas o seu mais importante papel deve ser associado à exploração de opções de manejo e alternativas silviculturais. Segundo Passioura (1996), os modelos descritivos podem ser chamados de modelos de engenharia (engineering models) e as melhores formulações são baseadas em relações empíricas entre o comportamento da planta e as principais variáveis independentes consideradas. $E$ acrescenta que, em função dessa natureza empírica, não se pode esperar sucesso em aplicações para fora da base de dados originais. Burkhart (1997) assinala que experiências com ajuste de modelos para diferentes bases de dados têm demonstrado que algumas formas funcionais são robustas (e assim apresentam certa generalidade). Mas aquelas equações muito complexas ajustadas por técnicas de regressão não linear e que, não consideram as bases conceituais do fenômeno sendo descrito, raramente apresentarão bons resultados contra novos dados.

Com base nessas afirmações, pode-se afirmar que uma boa formulação de modelos de crescimento descritivos necessita estar embasada numa amostragem suficientemente representativa da população de interesse e de modelos matemáticos robustos que considerem o fenômeno básico sendo tratado, assegurando maior generalidade para aplicação posterior. Os processos de desenvolvimento, avaliação e uso de modelos de crescimento exigem, assim, dados para levantamento das hipóteses de trabalho e uma base independente para validação das funções ajustadas. Segundo Vanclay (1999), interpolações são sempre mais seguras que extrapolações, o que sugere que os dados cubram a mais ampla faixa de situações em termos de capacidades produtivas e condições do povoamento. Dados limitados, mas confiáveis, em cada extremo e na região mediana, são mais indicados que uma concentração abundante em torno da média. Remedições são necessárias para detectar o crescimento e mudanças e devem cobrir uma faixa de tempo suficiente para incorporar variações climáticas. $O$ intervalo de medição deve ser longo o suficiente para possibilitar a diferenciação dos padrões de crescimento, e essas diferenças, devem ser superiores aos eventuais erros de medição. Os passos para a construção de ferramentas de inferência do crescimento florestal dentro de um processo de simulação, segundo Jones et al. (1998) são: definição dos objetivos, definição do sistema, análise de dados e revisão de literatura, desenvolvimento do modelo, implementação computacional verificação, calibração e validação do modelo, análise de sensitividade e aplicação do modelo.

As metodologias de modelagem estão em contínuo desenvolvimento e tornando-se mais sofisticadas à medida que novos fundamentos biológicos, técnicas estatísticas sofisticadas e tecnologia computacional mais potente são agregadas ao processo. Por outro lado, Levins (1966) argumenta que as três propriedades mais desejáveis de um modelo são: generalidade, realidade e precisão. Em qualquer modelo uma das propriedades não consegue ser totalmente atendida, mas alcançam alto nível de aceitação nas outras duas. Burkhart (1997) afirma que nos modelos de crescimento e produção tradicionais, a generalidade é sacrificada em detrimento das demais características, com maior ênfase em precisão. Dado os objetivos usuais atribuídos a esses modelos, essa é uma estratégia razoável. Contudo, devido às mudanças relativamente rápidas no manejo e nas condições ambientais, há um crescente interesse em intensificar a generalidade des- 
MAESTRI, R. et al. Modelagem do crescimento...

sas ferramentas. Para isso, é necessário integrar diferentes abordagens no desenvolvimento dos modelos e, aqueles que considerem um comportamento baseado em hipóteses biológicas, apresentam maior generalidade e utilidade, inspirando maior confiança por parte dos usuários. A literatura apresenta alguns exemplos de integração dessas diferentes abordagens mencionadas por Burkhart (1997) e que se baseiam em duas filosofias principais: a) ligação de modelos descritivos com modelos processuais através do desenvolvimento e identificação de um ponto em comum (Baldwin et al. (1993), Battaglia et al. (1999), Kimmins et al (1999), Almeida et al. (2002)); b) inclusão de variáveis ambientais na própria formulação matemática dos modelos. Dentro desse item, alguns trabalhos procuraram estimar o índice de sítio pelo uso de variáveis do ambiente (Hunter \& Gibson (1984), Carter et al. (1989), Kayahara et al. (1998), Curt et al. (2001)), enquanto outros procuraram estimar a produção volumétrica considerando as condições ambientais na formulação do modelo (Nautival \& Couto (1984), Woolons et al. (1997), Snowdon et al. (1998) e Snowdon et al. (1999), Robert (2003)).

A estimativa do índice de sítio via variáveis do ambiente apresentados na literatura científica, prima pela condição de pura descrição das relações entre a altura dominante numa determinada idade com variáveis de solo, clima e fisiografia. Os modelos desenvolvidos apresentam-se sempre na forma de modelo linear múltiplo, sem consideração de uma hipótese biológica que assegure maiores extrapolações. Outra forma de consideração das variáveis ambientais em modelos descritivos trata da suas inclusões diretamente no modelo de área basal ou volume por hectare (Castro et al. 2005). Alguns desenvolvimentos resultaram em modelos lineares múltiplos sem um maior embasamento biológico e outros tratam a questão de forma um pouco mais sofisticada. O melhor exemplo observado até o momento na literatura científica, trata-se do trabaIho de Snowdon et al. (1999), os quais incorporaram um índice climático dentro de modelo de crescimento para Pinus radiata na Nova Zelândia. Tanto a precipitação pluviométrica anual como a estimativa de carbono fixado obtido pelos modelos mecanísticos BIOMASS (McMurtrie et al., 1990) e GROWEST (Fitzpatrick \& Nix, 1970), foram testadas. Segundo esses autores, cada observação anual de um índice climático $\left(\mathrm{Cl}_{\mathrm{i}}\right)$ pode ser considerada como composta de duas partes: uma média histórica $(\mathrm{MH})$ e um desvio anual $\left(D_{i}\right)$ deste valor, ou seja, $\mathrm{Cl}_{i}=\mathrm{MH}+\mathrm{D}_{\mathrm{i}}$. Esses termos podem ser transformados em escala dividindo-se pela média histórica $(\mathrm{MH})$, resultando num índice climático escalar $\left(\mathrm{SCl}_{\mathrm{i}}\right)$, ou seja: $\mathrm{SCl}_{\mathrm{i}}=1+\mathrm{D}_{\mathrm{i}} / \mathrm{MH}$. A soma dos índices, desde o plantio até certa idade é obtida por: $S S C_{i}=S\left(1+D_{i} / M H\right)=$ idade $+S D_{i} /$ $\mathrm{MH}$ ) e, dentro da base de dados usada para definir a média histórica $(\mathrm{MH})$ os desvios escalares tem média zero e sua soma tende a zero. A formulação empírica escolhida para predizer o crescimento e a produção foi o modelo log-recíproco modificado de
Schumacher (Schumacher 1939; Clutter, 1963; Clutter \& Jones, 1980), dado por:

$$
\begin{aligned}
& Y_{2}=\exp \left(\operatorname{LOG}\left(\left(Y_{1}\right)\left(T_{1} / T_{2}\right)^{b}+a\left(1-\left(T_{1} / T_{2}\right)^{b}\right)\right)\right. \\
& \text { onde } Y_{1}, Y_{2} \text { representam a produção atual }
\end{aligned}
$$

e futura correspondentes às idades $T_{1}, T_{2}$ respectivamente, a é a estimativa do parâmetro de máxima produção (assíntota) e b o parâmetro de forma. $\mathrm{Na}$ formulação original da equação de Schumacher, a era uma função do índice de sítio, b assumia valor igual a 1 e $\mathrm{T}$ era idêntico à idade. Esses autores analisaram diversas formas de inclusão dos desvios escalares nos termos a e T, substituindo-os por funções contendo tais índices climáticos derivados do modelo mecanístico BIOMASS e que permitiram reduções superiores a $50 \%$ no erro da estimativa em área basal.

\section{PROPOSTA DE MODELOS DESCRITIVOS COM VARIÁVEIS AMBIENTAIS}

Conforme Maestri (2003), alguns estudos tem sido desenvolvidos com base na inclusão de variáveis ambientais nas formulações de modelos empíricos. Complementa ainda que existem duas principais abordagens, sendo: a) estimativa do índice de sitio a partir desta variável; b) estimativa da produção esperada a partir de variáveis ambientais e do povoamento.

O trabalho de Hunter \& Gibson (1984) citados por Maestri (2003), com a utilização da técnica de componentes principais, permitiu selecionar variáveis edáficas e climáticas que interferiam no crescimento. Estes autores observaram uma relação positiva entre índice de sítio, precipitação, nutrientes, profundidade de solo e penetrabilidade do solo para plantios de Pinus radiata na Nova Zelândia.

Conforme o trabalho de Carter et al. (1989) citados por Maestri (2003), foi possível relacionar índice de sitio com déficit de água no solo durante a estação de crescimento e a concentração de nitrogênio mineralizável no solo de povoamentos de Douglas-Fir na região de British Columbia, no Canadá.

Conrona et al. (1998) citados por Maestri (2003), desenvolveu um trabalho na província de Firenze na Itália, estudando índice de sitio e sua relação com fatores ambientais, observou significativa influência da precipitação pluviométrica, excesso de água no solo, teor de argila, conteúdo de carbonato de cálcio e fisiografia. Segundo este autor, cerca de $58 \%$ da variação observada no índice de sitio foi explicada pelas variáveis, num modelo de regressão linear múltipla.

Maestri (2003) cita o trabalho de Kayahara et al. (1998) investigaram que a relação entre índice de sítio e nutrientes do solo e das folhas de Tsuga heterophylla na costa sul da região de British Columbia no Canadá. Embora as medidas de solo e nitrogênio foliar tenham se mostrado importantes como variáveis explanatórias, a maior produtividade dos povoamentos não foi explicada pela maior disponibilidade de nutrientes.

Soares (1999) citado por Temps (2005) adicionou a precipitação anual no modelo de área 
basal, dentro do modelo de volume, obtendo resultados satisfatórios em sua pesquisa com Eucalyptus urophylla no estado da Bahia, Brasil.

Temps (2005) em seu trabalho com adição da precipitação pluviométrica na modelagem do crescimento e da produção florestal em povoamentos não desbastados de Pinus taeda L., concluiu que a inclusão de variáveis climáticas nos modelos biométricos tradicionais pode aumentar a precisão de inferência das variáveis biométricas, reduzindo o erro padrão da estimativa.

Tendo como premissa que um modelo descritivo aumenta sua capacidade generalista quando aspectos ambientais são incorporados em sua formulação e, adicionalmente, que o comportamento funcional do modelo deva se sustentar em bases biológicas, uma metodologia que considere essas duas situações pode representar um passo no avanço da modelagem florestal já apontado por diversos autores (Mohren \& Burkhart, 1994; Amaro \& Tomé, 1997; Reed, 1997; Burkhart, 1997).
A altura das árvores dominantes de um povoamento florestal representa a melhor expressão da capacidade do sítio. Sua modelagem, tradicionalmente, ocorre através do relacionamento desta variável com a idade e diversas classes de sítio são arbitradas, originando famílias de curvas de altura dominante (Maestri et al., 1995).

Como a altura dominante (Sítio) interfere em todo o procedimento de inferência posterior modelo de mortalidade (Rossi et al. 2006; Wyckoff \& Clark, 2002; Yang \& Titus, 2002; Zhao et al. 2004), área basal e volume, a inclusão de variáveis ambientais somente dentro de sua formulação, mantendo o restante do sistema de prognose de produção inalterado, resguarda a concepção original da modelagem descritiva (sítio é a expressão da capacidade produtiva), porém dentro de um novo conceito: o de modelo híbrido. O modelo de Chapman \& Richards possui características típicas do crescimento de qualquer variável biológica, traduzido pelo significado de seus parâmetros, conforme apresenta a Figura 1.

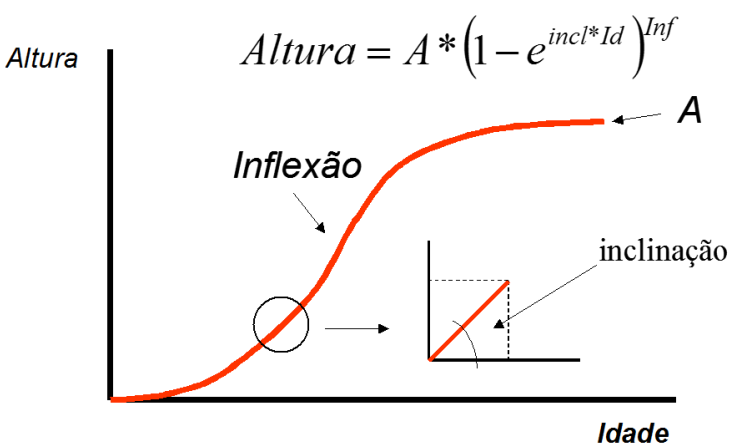

Figura 1 - Modelo biológico de Chapman \& Richards e o significado de seus coeficientes.

O coeficiente A do modelo de Chapman \& Richards representa o valor assintótico, ou seja, a máxima capacidade de produção e que tende a se estabilizar com o avanço da idade. O coeficiente de inclinação representa o ritmo de crescimento num determinado momento da vida do povoamento. O coeficiente de inflexão identifica a passagem de um ritmo acelerado de crescimento para um ritmo mais lento e aponta para o máximo incremento corrente.

São duas as formas de uso da função de Chapman \& Richards dentro de um sistema de crescimento e produção descritivo: a) para projetar o incremento a partir de uma informação inicial conhecida (inventário base numa determinada idade); b) para predizer as curvas dos diversos Índices de Sítio. No primeiro caso, uma transformação algébrica do modelo é necessária, tomando a seguinte forma, segundo Scolforo (1998):

$$
H d_{2}=A\left(\frac{H d_{1}}{A}\right)^{\frac{L n\left(1-e^{\left.i n c l . I d_{2}\right)}\right)}{\operatorname{Ln}\left(1-e^{i n c l . l d_{1}}\right)}}
$$

onde $\mathrm{Id}_{1}$ e $\mathrm{Hd}_{1}$ representam a idade e a altura dominante observadas e $\mathrm{Id}_{2}$ é a idade em que se deseja estimar a altura dominante $\mathrm{Hd}_{2}$. No segundo caso, o modelo permanece como demonstrado na Figura 1.

O incremento em altura dominante no modelo de Chapman \& Richards está relacionado ao coeficiente de inclinação da equação, conforme apresentado na Figura 1. Com base nesse princípio, um modificador do coeficiente de inclinação, a partir de uma composição das variáveis ambientais, permite a consideração dos seus efeitos, mas preservando as características biológicas do modelo.

$$
\operatorname{Modi}_{i n c l}=d_{1} * \operatorname{var} a m b_{1}+d_{2} * \operatorname{var} a m b_{2}+\ldots .
$$


MAESTRI, R. et al. Modelagem do crescimento...

e sua inclusão no modelo obedece a seguinte forma:

$$
H d_{2}=A\left(\frac{H d_{1}}{A}\right)^{\frac{\operatorname{Ln}\left(1-e^{\left.i n C l "\left(M O D I_{i n c l}\right)^{*}+d_{2}\right)}\right)}{\operatorname{Ln}\left(1-e^{i n c l "\left(M O D I_{\text {incl }}\right)^{*}\left[d_{1}\right.}\right)}}
$$

onde Modi ${ }_{\text {ncl }}$ é um fator modificador do coeficiente de inclinação, var $a m b_{i}$ são as variáveis ambientais selecionadas e $\mathrm{d}_{1}, \mathrm{~d}_{2}$, os coeficientes da regressão.

Já para estimar Índice de Sítio e as curvas de evolução da altura dominante, um fator modificador em função de variáveis ambientais, deve ser associado ao coeficiente assintótico do modelo de Chapman \& Richards, tomando a seguinte forma:

$$
\operatorname{Modi}_{A}=c_{1} * \operatorname{var} a m b_{1}+c_{2} * \operatorname{var} a m b_{2}+\ldots .
$$

e sua inclusão no modelo obedece a seguinte forma:

$$
H d o m=A^{*}\left(\operatorname{Modi}_{A}\right) *\left(1-e^{i n c l * I d}\right)^{\text {inf }}
$$

onde Hdom é a altura dominante estimada para a idade $l d$ que é a idade em anos, $\mathrm{Modi}_{A}$ é o fator modificador do coeficiente assintótico em função das variáveis ambientais - var $a m b_{i}$ e, finalmente, $c_{1}, c_{2}$, são os coeficientes da regressão.

Um passo importante no desenvolvimento de modelos com essa característica é o pareamento correto dos dados das medições de cada parcela de inventário florestal com os dados das variáveis ambientais (Köhler et al., 2005). No caso do modelo de incremento em altura, subentende-se que as medições inicial e final (idades $\quad \mathrm{Id}_{1}$ e $\quad \mathrm{Id}_{2}$ ) da parcela permanente determinam o exato período para cômputo das variáveis ambientais associadas. No caso do modelo para índice de sítio, o intervalo de tempo considerado deve ir da data de plantio até a data da medição da altura dominante. A escolha das variáveis ambientais deve obedecer aos princípios básicos de relacionamento de dados. No caso do modelo do incremento em altura, devem ser selecionadas as variáveis que mais expliquem o crescimento anual daquela característica dos povoamentos. Para o modelo de índice de sítio, as variáveis ambientais devem demonstrar correlação com as alturas dominantes em diversas idades $\mathrm{e}$ serem selecionadas em função disso.

O sistema de produção e crescimento híbrido, assim desenvolvido, permite a atualização de levantamentos (inventários florestais) de forma bem mais precisa. Para isso, os modelos com fator modificador do coeficiente de inclinação podem ser usados, supondo que as variáveis ambientais foram também coletadas no período de interesse. Em locais sem histórico de plantios, onde não existe qualquer embasamento biométrico para estimativa da produtividade potencial, os modelos com fator modificador do coeficiente assintótico, são os recomendados e, as variáveis ambientais devem ser baseadas na média histórica do local. Os modelos híbridos em questão possibilitam o levantamento de suposições sobre como o ambiente interfere no crescimento, mas a identificação de lacunas de conhecimento das relações fisiológicas e ambientais com a produção para direcionamento de pesquisas deve ser feita com reservas, já que não se trata de modelagem dos "processos".

O volume é obtido, então, por uma sequência de projeções das características dos povoamentos, iniciando pela estimativa da altura dominante com base nas variáveis ambientais. Em seguida procede-se com a estimativa do número de árvores por hectare, da área basal e, finalmente, do volume (por árvore, estrutura diamétrica ou por hectare, através de modelos para árvore individual (Phillips et al., 2003), baseados em distribuições probabilísticas ou modelo global, respectivamente). Mas as variáveis ambientais somente incidirão no modelo de altura dominante, alterando assim, o conceito biométrico tradicional da inflexibilidade do índice de sítio para cada local. Isso é particularmente perceptível e válido para espécies de crescimento rápido, rotação curta e sensibilidade aos aspectos ambientais.

\section{CONSIDERAÇÃO FINAL}

A evolução da modelagem florestal
biométrica em função de novas técnicas 
MAESTRI, R. et al. Modelagem do crescimento...

estatísticas e do avanço da tecnologia da computação é facilmente percebida no meio científico nos últimos 30 anos. Por sua vez, a nova linha de abordagem do crescimento em função dos processos básicos que o regem, impulsionou os desenvolvimentos dos modelos mecanísticos principalmente na última década. Cada uma dessas filosofias apresenta características específicas levando a situações de maior ou menor aplicabilidade das ferramentas. Genericamente, como almejam objetivos um tanto quanto distintos, pode-se considerar que as duas correntes são complementares e não conflitivas.

Do ponto de vista dos modelos descritivos, a inclusão de variáveis do ambiente em sua formulação, permite maior explicação da variabilidade encontrada, a qual sempre foi absorvida no erro estatístico. Assim, além do aumento da precisão da estimativa, essa nova abordagem possibilita a flexibilização do conceito de Índice de Sítio, assumido historicamente como imutável para um determinado local dentro de uma rotação florestal. A observação prática tem demonstrado que espécies de acelerado ritmo de crescimento e de rotação curta apresentam oscilações de produtividade altamente correlacionada aos aspectos ambientais. E a inclusão dessas novas variáveis dentro da formulação matemática de modelos com comportamento funcional biológico, assegura maior capacidade generalista dos modelos descritivos.

\section{REFERÊNCIAS}

1. ALMEIDA, A C.; MAESTRI, R.; LANDSBERG, J.J.; SCOLFORO, J.R.S. Linking process-based and empirical forest models in Eucalyptus plantation in Brazil. In: Workshop Reality, Models and Parameter Estimation - The Forest Scenario, Portugal, 2002.

2. AMARO, A.; TOMÉ, M. Empirical and process based models for forest tree and stand growth simulation. Preface. Edições Salamandra, Oeiras, Portugal, pp. 5-7. 1997.

3. BALDWIN, V. C.; BURKHART, H. E.; DOUGHERTY, P. M.; TESKEY, R. O. Using a growth and yield model (PTAEDA2) as a driver for a biological process model (MAESTRO). U.S. Department of Agricultural, Forest Service, Southern Forest Experiment Station. Research Paper SO-276. 9pp. 1993.

4. BATTAGLIA, M.; SANDS, P.J.; CANDY, S.G. Hybrid growth model to predict height and volume growth in young Eucalyptus globulus plantations. Forest Ecology and Management, v. 120, n. 1, p.193-201. 1999.

5. BURKHART, H.E. Development of empirical growth and yield models. In: Amaro, A. and Tomé, M. (eds.). Empirical and process-based models for forest tree and stand growth simulation. Edições Salamandra, Oeiras, Portugal, pp. 53-60. 1997.

6. CARTER, R.E.; KLINKA, K. Relations between growing-season soil water-deficit, mineralizable soil nitrogen and site index of coastal Douglas-Fir. Forest Ecology and Management, v. 30, n. 1-4, pp. 301-311. 1989.

7. CASTRO MARÍN, G.; NYGARD, R.; GONZALES RIVAS, B.; ODEN, P. C. Stand dynamics and basal area change in a tropical dry forest reserve in Nicaragua. Forest Ecology and Management. v. 208, n. 1-3, p. 63-75, 2005.

8. CLUTTER, J.L., JONES, E.P. Prediction of growth after thinning in old-field slash pine plantations. USDA Forest Service Research. Paper SE-217. 14p. 1980.

9. CLUTTER, J.L., FORTSON, J.C., PIENAAR, L.V., BRISTER, G.H., BAILEY, R.L. Timber management: a quantitative approach. New York, John Wiley \& Sons, 1983. 333p.

10. COMINS, H.N.; MCMURTRIE, R.E. Long-term response of nutrient-limited forests to $\mathrm{CO}_{2}$ enrichment; equilibrium behavior of plant-soil models. Ecological Applications, v. 3, n.4, pp. 666-681. 1993.

11. CURT, T.; BOUCHAUD, M.; AGRECH, G. Predicting site index for Douglas-fir plantations from ecological variables in the Massif Central area of France. Forest Ecology and Management, v. 149, n.1, pp. 61-74. 2001.

12. FITZPATRICK, E.A.; NIX, H.A. The climatic factor in Australian grassland ecology. In: Moore, R.M., (Ed.), Australian Grasslands, Australian National University Press, Canberra. 1970.

13. HUNTER, I.R.; GIBSON, A.R. Predicting Pinus radiata site index from environmental variables. New Zealand Journal of Forestry Science, v. 14, n.1, pp. 53-64. 1984.

14. JONES, J.W.; MISHOE, J.W.; BOETE, K.J. Introduction on simulation and modeling. Food \& Fertilizer Technology Center. v.4, n. 13. pp. 3-21. 1998.

15. KAYAHARA, G.; CARTER, R.E.; KLINKA, K. Site index of western hemlock (Tsuga heterophylla) in relation to soil nutrient and foliar chemical measures. Forest Ecology and Management, v. 74, n.1-3, pp. 161-169. 1998.

16. KIMMINS, J.P.; SCOULLAR, K.A.; SEELY, B.; ANDISON, D.W.; BRADLEY, R.; MAILLY, D.; TSZE, K.M. Forceeing and forecasting the horizon: hybrid simulation modeling of forest ecosystem sustainability. In: Amaro, A. and Tomé, M. (eds.) Empirical and process based models for forest tree and stand growth simulation. Edições Salamandra, Oeiras, Portugal, v. 1. pp. 431-441. 1999.

17. KÖHLER, P.; DITZER, T.; ONG, R. C.; HUTH, A. Comparison of measured and modelled growth on permanent plots in Sabahs rain forests. Forest Ecology and Management. v. 144, n. 1-3, p.101-111, 2001

18. LANDSBERG, J.J.; WARING, R.H. A generalised model of forest productivity using simplified concepts of radiationuse efficiency, carbon balance and partitioning. Forest Ecology and Management, v. 95, n.3, pp. 209-228.1997.

19. LEVINS, R. The strategy of model building in population biology. American Scientist, v. 54, n. 1. p.421-431. 1966.

20. MAESTRI, R.; SCOLFORO, J.R.S.; HOSOKAWA, R.T. Um sistema de predição do crescimento e da produção para povoamentos de acácia-negra (Acacia mearnsii de Wild). Revista Árvore, v. 19, n. 3, pp. 358-381. 1995.

21. MAESTRI, R. Modelo de crescimento e produção para povoamentos clonais de Eucalyptus grandis considerando variáveis ambientais. Tese de Doutorado do programa de Pós-Graduação em Engenharia Florestal da Universidade Federal do Paraná. 2003. 142p.

22. MCMURTRIE, R.E.; ROOK, D.A.; KELLHER, F.M. Modelling the yield of Pinus radiata on a site limited by water and nitrogen. Forest Ecology and Management, v. 30, n. 3, pp.381-413. 1990. 
MAESTRI, R. et al. Modelagem do crescimento...

23. MOHREN, G.M.J.; BURKHART, H.E. Contrasts between biologically-based process models and managementoriented growth and yield models. Forest Ecology and Management. v. 69, n. 1. pp. 1-5.1994.

24. NAUTIVAL, J.C.; COUTO, L. The nature and uses of the timber production function: Eucalyptus grandis in Brazil. Forest Science. v. 30. n. 3. pp. 761-773. 1984

25. PASSIOURA, J.B. Simulation models: science, snake oil, education, or engineering? Agronomy Journal, v. 88 , n. 2. pp. 690-694. 1996.

26. PHILLIPS, P. D.; BRASH, T. E.; YASMAN, I.; SUBAGYO, P.; GARDINGEN, P. R. An individual based spatially explicit tree growth model for forests in East Kalimantan (Indonesian Borneo). Ecological Modelling. v. 159, n. 1, p. 1-26, 2003.

27. REED, D. D. Ecophysiological models of forest growth: uses and limitations. In: Amaro, A.; Tomé, M. (eds.). Empirical and Process-based models for Forest Tree and Stand Growth Simulation. Edições Salamandra, Oeiras, Portugal, pp. 305-311. 1997.

28. ROBERT, A. Simulation of the effect of topography and tree falls on stand dynamics and stand structure tropical forests. Ecological Modelling. v. 167, n. 3, p. 287-303, 2003.

29. ROSSI, L.M.B.; KOEHLER, H.S.; SANQUETTA, C.R.; ARCE, J.E. Modelagem de Mortalidade em Florestas Naturais. Floresta. v. 37, n. 2, 275-291p. 2007.

30. SCHUMACHER, F.X. A new growth curve and its application to timber-yield studies. Journal of Forestry, v. 37, n.1, p. 819-820. 1939.

31. SCOLFORO, J. R. S. Modelagem do Crescimento e da Produção de Florestas Plantadas e Nativas, UFLA/FAEPE, Lavras, 441p. 1998.

32. SNOWDON, P., WOOLLONS, R.C.; BENSON, M.L. Incorporation of climatic indices into models of growth of Pinus radiata in a spacing experiment. New Forest, v. 16, n.1, pp. 101-123. 1998.

33. SNOWDON, P.; JOVANOVIC, T.; BOOTH, T.H. Incorporation of indices of annual climatic variation into growth models for Pinus radiata. Forest Ecology and Management, v. 117, n.1-3, pp. 187-197. 1999.

34. TEMPS, M. Adição da precipitação pluviométrica na modelagem do crescimento e da produção florestal em povoamentos não desbastados de Pinus taeda L. Dissertação de Mestrado do programa de Pós-Graduação em Engenharia Florestal da Universidade Federal do Paraná. 2005. 83p.

35. VANCLAY, J. K. Modelling Forest Growth and Yield - Applications to Mixed Tropical Forests, CABI Publishing, Oxon, UK, 312. 1999.

36. WYCKOFF, P. H.; J. S. The relationship between growth and mortality for seven co-occurring tree species in the southern Appalachian Mountains. Journal of Ecology. v. 90, n. 4, p. 604-615, 2002.

37. WOOLLONS, R.C.; SNOWDON, P.; MITCHELL, N.D. Augmenting empirical stand projection equation with edaphic and climatic variables. Forest Ecology and Management, v. 98, n.1, pp. 267-275.1997.

38. YANG, Y.; TITUS, S. J. Maximum size-density relationship for constraining individual tree mortality functions. Forest Ecology and Management. v. 168, n. 1-3, p. 259-273, 2002.

39. ZHAO, D.; BORDERS, B.; WILSON, M. Individual-tree diameter growth and mortality models for bottomland mixedspecies hardwood stands in the lower Mississippi alluvial valley. Forest Ecology and Management. v. 199, n. 2-3, p. 307-322, 2004.

Recebido em 23/03/2011

Aceito em 12/10/2013 